Estudios Geológicos, 66(1)

enero-junio 2010, 83-90

ISSN: $0367-0449$

doi:10.3989/egeol.40133.090

\title{
Reactividad potencial de las rocas andesíticas de Cabo de Gata (SE de España)
}

\section{Potential reactivity of the andesitic rocks from Cabo de Gata (SE Spain)}

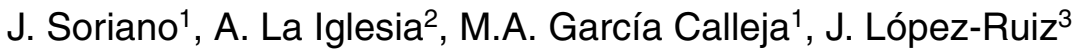

RESUMEN

Las rocas andesíticas producen una reacción álcalis-sílice con los componentes de la fase intersticial del hormigón. Esta reacción puede encuadrarse dentro de las de cinética lenta, ya que la disolución incongruente de los minerales constituyentes de las rocas, da lugar a la formación tardía de geles silicocalco-alcalinos. En consecuencia, la utilización convencional del método de ensayo de barras de mortero no detecta la reacción hasta transcurridos al menos 90 días.

Palabras clave: áridos, andesitas, reacción álcalis-sílice, reactividad potencial, Cabo de Gata.

\section{ABSTRACT}

Andesitic rocks produce an alkali-silica reaction with the components of the interstitial phase of concrete. This reaction can be considered as belonging to the slow-kinetic type, since the incongruent dissolution of the rock-forming minerals leads to the late formation of siliceous calc-alkaline gel. As a conquence, the conventional mortar-bar method does not detect such reaction until 90 days later.

Key words: aggregates, andesites, alkali-silica reaction, potential reactivity, Cabo de Gata.

\section{Introducción}

La durabilidad de un material se puede definir como la capacidad de mantener un adecuado comportamiento, propiedades y condiciones de seguridad, durante el tiempo y las condiciones de servicio previstas para su empleo. En el caso del hormigón, la durabilidad es la capacidad para resistir ataques físicos, fisicoquímicos y químicos tanto de origen interno como externo (ACI Committee 201, 2007).

Cuando hay que evaluar la influencia ejercida por diversos factores en la durabilidad del hormigón nos encontramos con el grave problema de la importancia relativa que los diferentes autores prestan a cada uno de los factores indicados. Así, los de formación más técnica tiende a conceder mayor importancia a los aspectos mecánicos y físicos que a los químicos y fisicoquímicos. Por el contrario, los de formación más científica tienden a considerar principalmente los factores de tipo químico o fisicoquímico tales como composición del cemento, reactividad de los áridos, etc.

Algunos áridos de naturaleza silícea pueden tener una elevada reactividad potencial, ya que reaccionan con los álcalis de la fase intersticial del hormigón, dando lugar a la formación de geles y compuestos de sílice. En general, estos compuestos tienen capacidad para generar en presencia de agua una elevada presión osmótica, lo que da lugar a un aumento de volumen y a importantes presiones disruptivas.

\footnotetext{
${ }^{1}$ Laboratorio Central de Estructuras y Materiales. CEDEX-Ministerio de Fomento. Alfonso XII, 3. 28014 Madrid (jsoriano@cedex.es) (magarcia@cedex.es).

2 Departamento de Petrología y Geoquímica, Instituto de Geología Económica, CSIC-UCM. Ciudad Universitaria. 28040 Madrid (iglesia@geo.ucm.es).

3 Departamento de Geología, Museo Nacional de Ciencias Naturales, CSIC. José Gutiérrez Abascal, 2. 28006 Madrid (lopezruiz@mncn.csic.es).
} 
El esquema general de este mecanismo es el siguiente:

$$
\begin{gathered}
\mathrm{n}_{3} \mathrm{SiO}_{2}+\mathrm{n}_{1 / 2} \mathrm{NaOH}+\mathrm{n}_{2} \mathrm{Ca}(\mathrm{OH})_{2}+\mathrm{n}_{4} \mathrm{H}_{2} \mathrm{O} \Rightarrow \\
\Rightarrow \mathrm{n}_{1} \mathrm{Na}_{2} \mathrm{O} \cdot \mathrm{n}_{2} \mathrm{CaO} \cdot \mathrm{n}_{3} \mathrm{SiO}_{2} \cdot \mathrm{n}_{4} \mathrm{H}_{2} \mathrm{O}
\end{gathered}
$$

Según la práctica general de la química del cemento, el $\mathrm{NaOH}$ representa al $\mathrm{Na}_{2} \mathrm{O}$ equivalente (es decir, $\mathrm{Na}_{2} \mathrm{O}+0,66 \mathrm{~K}_{2} \mathrm{O}$ ). A su vez, el gel formado será o no de carácter expansivo en función del contenido en $\mathrm{CaO}$.

La sílice de las rocas y minerales susceptibles de ser utilizados como áridos reactivos, interacciona con el $\mathrm{NaOH}$ produciendo, en primer lugar, una reacción ácido-base entre los grupos silanol $\mathrm{Si}-\mathrm{OH}$ y los iones $\mathrm{OH}^{-}$de la solución alcalina, después de la neutralización por un ión $\mathrm{Na}^{+}$de la carga negativa formada. Posteriormente se produce una segunda reacción consistente en un ataque de puentes siloxanos por iones $\mathrm{OH}^{-}$, lo que provoca una destrucción de la estructura y el paso de la sílice a $\mathrm{SiO}_{4} \mathrm{H}_{2}=$. Hay que señalar que ambas reacciones se producen casi simultáneamente, dando lugar a la formación de un gel de silicato sódico de fuerte carácter expansivo.

En este trabajo se estudia la reactividad potencial de rocas andesíticas. Aunque es bien sabido que estas rocas pueden ser reactivas frente a los componentes de la fase instersticial del hormigón, sin embargo su cinética de reacción no es suficientemente conocida. Para abordar este último aspecto se han seleccionado rocas calco-alcalinas de la región volcánica de Cabo de Gata, en donde estos materiales están bien representados.

\section{La región volcánica de Cabo de Gata}

La región volcánica de Cabo de Gata se localiza en la parte oriental de la Cordillera Bética. Forma parte de una provincia más extensa, la denominada provincia volcánica del SE de España, que se extiende a lo largo de una banda que abarca desde el Cabo de Gata hasta el Mar Menor, aunque también pertenecen a ella los afloramientos de rocas ultrapotásicas (lamproítas) localizados más al interior, en el dominio externo de la Cordillera Bética (por ej., López-Ruiz et al., 2002).

Las rocas volcánicas de Cabo de Gata se pueden clasificar en andesitas basálticas, andesitas piroxénico-anfibólicas y dacitas. Esta clasificación está basada en criterios geoquímicos, como el contenido en $\mathrm{K}_{2} \mathrm{O}$ frente a $\mathrm{SiO}_{2}$, ya que los caracteres petrológico-mineralógicos de estas rocas no son los suficientemente diagnósticos como para permitir su correcta clasificación.

Observadas al microscopio las lavas andesíticobasálticas y andesíticas están constituidas esencialmente por grandes cristales de plagioclasa $\left(\mathrm{An}_{95-68}\right)$, ortopiroxeno $\left(\mathrm{En}_{79-55} \mathrm{Fs}_{18-43} \mathrm{Wo}_{3-2}\right)$ y clinopiroxeno $\left(\mathrm{En}_{50-41} \mathrm{Fs}_{9-17} \mathrm{Wo}_{41-42}\right)$, si bien en las más silíceas de estas últimas aparecen también cristales de anfíbol, biotita y cuarzo, que están incluidos en una matriz vítrea o poco cristalina que puede representar hasta el 50\% de la roca, en la que aparecen además magnetita $\left(\mathrm{Usp}_{29-11}\right)$ e ilmenita $\left(\mathrm{Ilm}_{80-74}\right)$ (fig. 1). Las lavas dacíticas presentan, además de plagioclasa $\left(\mathrm{An}_{90-67}\right)$ y ortopiroxeno $\left(\mathrm{En}_{58-55} \mathrm{Fs}_{40-43}\right.$ $\mathrm{Wo}_{2}$ ), proporciones mayores de anfíbol (hornblenda-cummingtonita), biotita y cuarzo, incluidos asimismo en una matriz vítrea o poco cristalina en la que cristalizan además magnetita $\left(\mathrm{Usp}_{26-21}\right)$ e ilmenita $\left(\mathrm{Ilm}_{80-73}\right)$ (fig. 2). Un detallado estudio de los aspectos mineralógicos y petrológicos de estas rocas se puede encontrar en López-Ruiz \& Rodríguez Badiola (1980).

En lo que concierne a su composición química, las rocas de Cabo de Gata exhiben un relativamente amplio rango de $\mathrm{SiO}_{2}$, así como altos contenidos de $\mathrm{Al}_{2} \mathrm{O}_{3}$ y CaO, moderados de $\mathrm{Na}_{2} \mathrm{O}$ y bajos de $\mathrm{K}_{2} \mathrm{O}$ y $\mathrm{P}_{2} \mathrm{O}_{5}$. Asimismo, presentan un típico enriquecimiento en elementos traza de elevado radio iónico (que es más alto en $\mathrm{Rb}$ y $\mathrm{Th}$ que en $\mathrm{Ba}$ ) y tierras raras ligeras y un característico empobrecimiento en $\mathrm{Sr}, \mathrm{Nb}$, Ta y Ti. Con el incremento del contenido en $\mathrm{SiO}_{2}$ (esto es, al pasar de las andesitas basálticas a las riolitas) se produce un progresivo enriquecimiento de $\mathrm{K}_{2} \mathrm{O}, \mathrm{Rb}$, Th, $\mathrm{Zr}$ y tierras raras, así como un empobrecimento en $\mathrm{FeO}, \mathrm{MgO}, \mathrm{CaO}, \mathrm{TiO}_{2}$, $\mathrm{P}_{2} \mathrm{O}_{5}, \mathrm{Co}$, Ni y V.

\section{Muestras estudiadas y métodos experimentales}

Se han seleccionado dos muestras representativas de la región de Cabo de Gata (figs. 1 y 2). Una andesita piroxénica de la Mesa de Roldán (AMR) y una dacita anfibólica de Punta Baja (DPB). La andesita piroxénica corresponde a una roca porfídica, constituida por abundantes fenocristales de plagioclasa y ortopiroxeno (y en menor proporción y de menor tamaño de clinopiroxeno y hornblenda), 


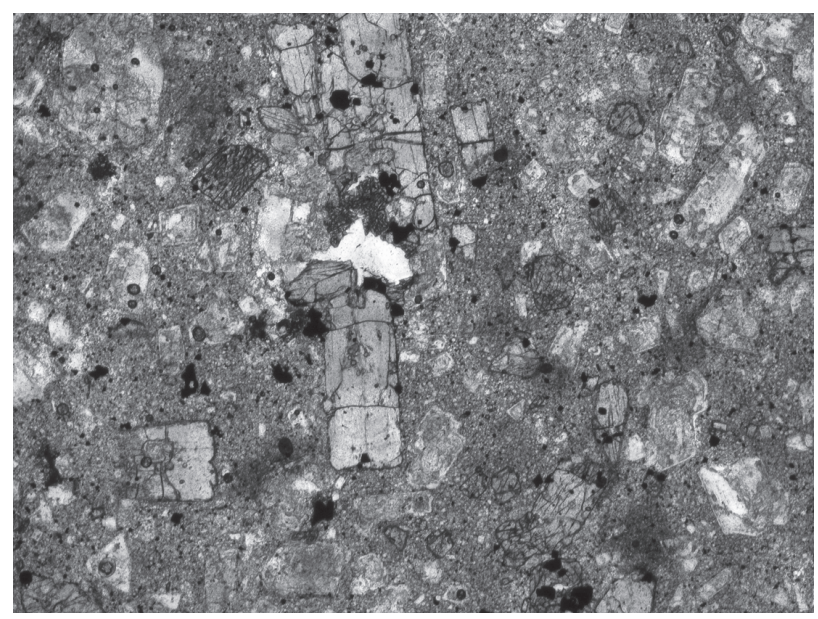

Fig. 1.-Andesita piroxénica (AMR). Fenocristales idiomorfos de ortopiroxeno (centro), clinopiroxeno (izq.) y plagioclasa (parcialmente sericitizados), en una matriz microcristalina en la que aparecen además cuarzo, magnetita e ilmenita.

incluidos en una matriz microcristalina constituida además por cuarzo, magnetita e ilmenita. Los cristales de plagioclasa están transformados en sericita, excepto su borde más exterior (que es menos cálcico) que está fresco. La dacita anfibólica corresponde, asimismo, a una roca porfídica, con abundantes fenocristales de plagioclasa, en menor porporción de hornblenda y biotita, y muy escasos de ortopiroxeno, incluidos en una matriz microcristalina constituida por los mismos minerales, además de cuarzo, magnetita e ilmenita. Los cristales de plagioclasa y de piroxeno están completamente alterados, y los de hornblenda muestran sus bordes oxidados.

La caracterizacion mineralógica de las muestras se llevó a cabo por microscopía óptica convencional y difracción de rayos X (DRX). En este último caso se utilizó un difractómetro Bruker D8 Advance provisto de un detector Sol-X. Para el análisis cuantitativo se empleó el método Chung (1975), usando software EVA de Bruker.

Para estudiar la reactividad potencial, las muestras se pulverizaron a un tamaño inferior a $75 \mathrm{mi}$ crones. $5 \mathrm{~g}$ de esta fracción se trataron con $40 \mathrm{ml}$ de disolución saturada de hidróxido cálcico o con disolución $1 \mathrm{~N}$ de hidróxido sódico. Este procedimiento es similar al utilizado por Way and Cole (1982) con pequeñas diferencias; así, la temperatura de reacción se mantuvo a $25^{\circ} \mathrm{C}$ en lugar de los $50{ }^{\circ} \mathrm{C}$ y el tiempo de reacción en 28 días en lugar de 40 días. Transcurrido ese tiempo se filtraron las disoluciones y se determinaron las concentraciones de $\mathrm{Si}, \mathrm{Al}, \mathrm{Ca}$, $\mathrm{P}, \mathrm{Na}$ y $\mathrm{K}$ en un espectrómetro ICP-OES Perkin

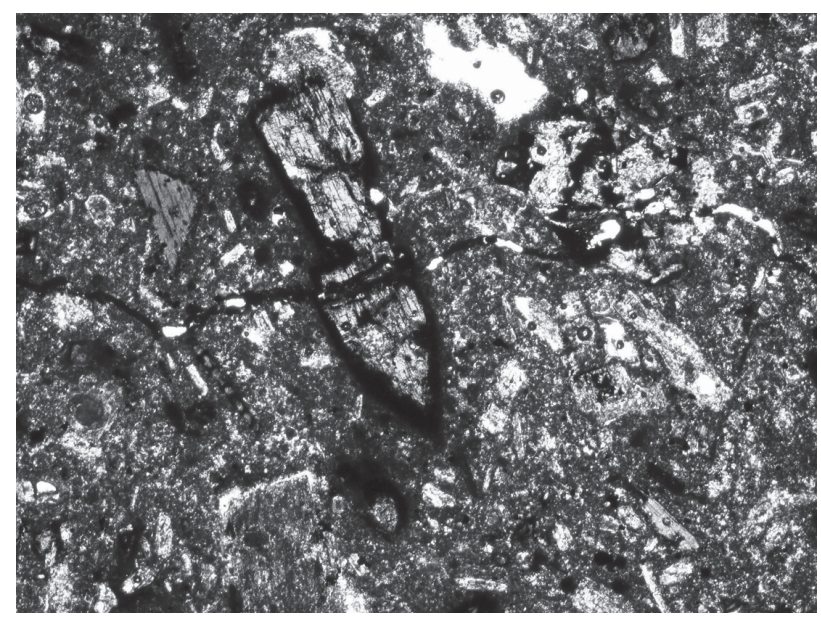

Fig. 2.-Dacita anfibólica (DPB). Fenocristales de anfíbol (con sus bordes oxidados), plagioclasa, biotita y cuarzo en una matriz intensamente alterada, constituida además por magnetita e ilmenita.

Elmer 4300DV. Los productos sólidos se lavaron dos veces con pequeñas cantidades de agua desionizada, se secaron en un desecador de $\mathrm{NO}_{3} \mathrm{Ca} 4 \mathrm{H}_{2} \mathrm{O}$ y se molieron y tamizaron hasta $53 \mathrm{~m} \mu$ para su estudio por microscopía electrónica de barrido (MEB), fluorescencia de rayos X (FRX) y (DRX).

La reactividad en superficie se estudió utilizando un microsocopio electrónico de barrido Zeiss EVO-50. De acuerdo con el método descrito por Soriano (1987), se emplearon pequeños fragmentos de roca de un peso próximo a los $5 \mathrm{~g}$, que se atacaron con $40 \mathrm{ml}$ de una solución $1 \mathrm{~N}$ de $\mathrm{NaOH}$.

La composición química de las muestras se estudió por FRX utilizando un espectrómetro Philips PW-1404 con tubo de Sc-Mo, detector de escintilación de gas PR-10, cristales analizadores de LiF, PE y TLAP, y software analítico Súper-Q Manager.

Finalmente, el ensayo acelerado en probetas de mortero para la determinación de la reactividad álcali-sílice se realizó de acuerdo con la norma UNE 146508, si bien el ensayo se prolongó hasta los 180 días.

\section{Resultados y discusión}

\section{Mineralogía}

La difracción de rayos $\mathrm{X}$ confirma la existencia de plagioclasa, cuarzo, enstatita y caolinita en las muestras AMR (natural y atacadas) y de plagioclasa, cuarzo, anfíbol, biotita y esmectita en las muestras DPB 
(tabla 1). Estos resultados indican que los tratamientos producen pocos cambios en la composición mineralógica de las muestras tratadas, ya que sólo se observa una ligera disminución en el contenido de anfíbol en la muestra DPB y de caolinita en la AMR. En ninguno de los difractogramas de las muestras tratadas aparecen nuevas líneas de difracción en el intervalo 7.9-7.6 ̊, como ocurría en las experiencias de Way and Cole (1985), ni en ningún otro intervalo. Tampoco se aprecia ensanchamiento de picos ni aparición de hombros que pudiera corresponder a la formación de nuevas fases cristalinas. Sin embargo, aparece un pequeño abombamiento de la línea base, entre 25 y $40^{\circ} 2 \theta(\mathrm{Cu} \mathrm{K} \alpha)$, que justificaría la presencia de geles en las muestras tratadas.

\section{Composición química}

En lo que concierne a la composición química de las andesitas y dacitas estudiadas (tabla 2), las abundancias de todos los elementos mayores es la típica de estas rocas, ya que presentan altos contenidos de $\mathrm{Al}_{2} \mathrm{O}_{3}$ y $\mathrm{CaO}$, moderados de álcalis y bajos de $\mathrm{TiO}_{2}$ y $\mathrm{P}_{2} \mathrm{O}_{5}$. El contenido en $\mathrm{K}_{2} \mathrm{O}$ de la dacita estudiada es anormalmente elevado, debido probablemente al intenso proceso de hidratación/desvitrificación que frecuentemente experimentan las rocas de esta región volcánica (López-Ruiz \& Wasserman, 1991).

El porcentaje de elementos mayores se modifica poco con el tratamiento, ya que sólo se aprecia una relativamente importante disminución de $\mathrm{MgO}$ en la muestra AMR y una ligera pérdida de $\mathrm{SiO}_{2}$ y $\mathrm{Al}_{2} \mathrm{O}_{3}$ en las muestras tratadas con $\mathrm{NaOH}$ (alrededor del $2 \%$ ) y en las tratadas con $\mathrm{Ca}(\mathrm{OH})_{2}$ (alrededor del $1 \%$ ). La variación en el contenido de $\mathrm{K}_{2} \mathrm{O}$ es similar, aunque la muestra AMR pierde más que la DPB.

En lo que concierne a los elementos traza, las variaciones más significativas se observan en el $\mathrm{Ba}$ y el Cr. El Ba se reduce del orden del $50 \%$ en la muestra tratada con $\mathrm{NaOH}$ y algo más en la tratada con $\mathrm{Ca}(\mathrm{OH})_{2}$. A su vez, la abundancia de $\mathrm{Cr}$ aumenta un $50 \%$ en la muestra tratada con $\mathrm{Ca}(\mathrm{OH})_{2}$ y algo menos en la tratada con $\mathrm{NaOH}$.

La disminución de los contenidos de $\mathrm{MgO}, \mathrm{SiO}_{2}$, $\mathrm{Al}_{2} \mathrm{O}_{3}$ y $\mathrm{Ba}$ se puede explicar por la disolución incongruente de los piroxenos, anfíboles y, en menor medida, de las biotitas de las rocas. Esta disolución da lugar a la formación de geles silicocalco-alcalinos, los cuales a su vez podrían actuar de cambiadores catiónicos, favoreciendo, por ejemplo, el enriquecimiento en $\mathrm{Cr}$.

\section{Composición química de los filtrados}

Las concentraciones de $\mathrm{Si}, \mathrm{Al}, \mathrm{Na}$ y $\mathrm{K}$ liberadas por los ataques alcalinos después de 28 días de tratamiento, así como el porcentaje de óxido liberado respecto al total de la muestra, se presentan en la tabla 3 . Estos resultados ponen de manifiesto que las concentraciones obtenidas son congruentes con los datos de FRX, tanto para las muestras naturales como para las tratadas. Por otra parte, la muestra AMR es más reactiva que la DPB. De la misma forma, las abundancias de $\mathrm{Si}$, Al y $\mathrm{K}$ liberadas en ambas muestras por la disolución de $\mathrm{Ca}(\mathrm{OH})_{2}$ son más bajas que las liberadas por la disolución de $\mathrm{NaOH}$. Las razones molares $\mathrm{SiO}_{2} / \mathrm{Al}_{2} \mathrm{O}_{3}$ en las disoluciones de $\mathrm{NaOH}$ son respectivamente 1,71 y 2,19 para las muestras DPB y AMR, lo que está de acuerdo con sus contenidos respectivos de caolinita y esmectita.

\section{Reactividad superficial}

El cambio de textura de los minerales de las muestras antes y después de ser sometidas a la acción de soluciones $1 \mathrm{~N}$ de $\mathrm{NaOH}$ o saturada de $\mathrm{Ca}(\mathrm{OH})_{2}$, pone de manifiesto que dichos minerales son susceptibles de reaccionar con los componentes de la fase intersticial del hormigón, lo que provoca la disolución parcial de aquéllos (fig. 3, 1-4).

\section{Sólidos obtenidos por evaporación}

El residuo sólido obtenido por evaporación hasta sequedad de los líquidos de ataque está constituido por geles silico-alumínico-calco-alcalinos, geles silico-calco-alcalinos, y compuestos polimorfos, semejantes a los encontrados en un hormigón afectado por una reacción álcali-sílice (fig. 3, 5-6).

\section{Barras de mortero}

La observación mediante MEB de la superficie de fractura de las barras de mortero indica que la expansión producida es debida a una reacción álcali-sílice. Así, se ha detectado la presencia de geles lisos (fig. 4, 1), geles rugosos (fig. 4, 2), agregados botroidales (fig. 4, 3), y productos polimorfos con texturas alveolar (fig. 4, 4), acicular (fig. 4, 5) y en pétalo de rosa (fig. 4, 6). 
Tabla 1.-Composición modal de las andesitas y dacitas naturales y tratadas, obtenida a partir de los análisis de DRX

\begin{tabular}{|c|c|c|c|c|c|c|c|}
\hline & Anfíbol & Plagioclasa & Biotita & Enstatita & Caolinita & Esmectita & Cuarzo \\
\hline AMR & - & 59 & - & 18 & 7 & - & 15 \\
\hline AMR-Ca & - & 61 & - & 19 & 7 & - & 14 \\
\hline AMR-Na & - & 61 & - & 19 & 5 & - & 15 \\
\hline DPB & 12 & 49 & 12 & - & - & 10 & 17 \\
\hline DPB-Ca & 11 & 49 & 13 & - & - & 11 & 16 \\
\hline DPB-Na & 8 & 51 & 14 & - & - & 10 & 17 \\
\hline
\end{tabular}

Tabla 2.-Composición química (elementos mayores en \% y elementos traza en ppm) de las andesitas y dacitas naturales y tratadas

\begin{tabular}{|c|c|c|c|c|c|c|}
\hline & AMR & $\mathrm{AMR}-\mathrm{Ca}(\mathrm{OH})_{2}$ & AMR-Na(OH) & DPB & $\mathrm{DPB}-\mathrm{Ca}(\mathrm{OH})_{2}$ & $\mathrm{DPB}-\mathrm{Na}(\mathrm{OH})$ \\
\hline $\mathrm{SiO}_{2}$ & 56,608 & 56,023 & 55,534 & 62,272 & 61,241 & 61,00 \\
\hline $\mathrm{Al}_{2} \mathrm{O}_{3}$ & 20,795 & 20,702 & 20,558 & 16,843 & 16,716 & 16,354 \\
\hline $\mathrm{Fe}_{2} \mathrm{O}_{3}$ (total) & 4,473 & 4,564 & 4,244 & 3,776 & 3,925 & 3,919 \\
\hline $\mathrm{MnO}$ & 0,113 & 0,102 & 0,091 & 0,071 & 0,083 & 0,085 \\
\hline $\mathrm{MgO}$ & 3,131 & 2,751 & 2,705 & 3,431 & 3,418 & 3,403 \\
\hline $\mathrm{CaO}$ & 3,604 & 4,748 & 3,713 & 1,512 & 2,361 & 1,574 \\
\hline $\mathrm{Na}_{2} \mathrm{O}$ & 1,936 & 1,812 & 3,862 & 1,705 & 1,377 & 2,539 \\
\hline $\mathrm{K}_{2} \mathrm{O}$ & 2,204 & 2,038 & 1,911 & 6,841 & 6,287 & 6,315 \\
\hline $\mathrm{TiO}_{2}$ & 0,484 & 0,418 & 0,392 & 0,508 & 0,497 & 0,512 \\
\hline $\mathrm{P}_{2} \mathrm{O}_{5}$ & 0,082 & 0,082 & 0,07 & 0,122 & 0,124 & 0,117 \\
\hline P.C. & 6,37 & 6,76 & 6,22 & 4,14 & 3,97 & 3,71 \\
\hline $\mathrm{Zr}$ & 71,3 & 67,3 & 81 & 144,1 & 139,2 & 119 \\
\hline Y & 13,3 & 10,6 & 14,4 & 12,9 & 13,4 & 12,1 \\
\hline $\mathrm{Rb}$ & 33,3 & 28,1 & 31,1 & 281,8 & 267 & 240,5 \\
\hline $\mathrm{Sr}$ & 165,5 & 155,9 & 184,3 & 94,0 & 95,7 & 85,3 \\
\hline Th & 2,3 & - & - & 7,6 & 11,8 & 19,9 \\
\hline $\mathrm{Nb}$ & 1,2 & 1,3 & 2,3 & - & 1 & 3,7 \\
\hline $\mathrm{Cu}$ & 10,4 & 13,9 & 14,8 & 1,9 & - & - \\
\hline $\mathrm{Ni}$ & 11,2 & 9,9 & 10,3 & 6,0 & 6 & 5,6 \\
\hline Co & 17,2 & 15,1 & 15,1 & 12,6 & 11,7 & 10,2 \\
\hline $\mathrm{Zn}$ & 43,6 & 36,1 & 37 & 56,2 & 50,4 & 51,1 \\
\hline $\mathrm{Pb}$ & 10,6 & 7,6 & 13 & 58,7 & 41,6 & 38,8 \\
\hline $\mathrm{Ce}$ & 25,9 & 26,1 & 35,6 & 41,9 & 50,1 & 37,9 \\
\hline $\mathrm{Cr}$ & 5,4 & 10,9 & 10 & 18,1 & 22,8 & 17,1 \\
\hline V & 117,5 & 108,5 & 96 & 91,4 & 81,4 & 82,5 \\
\hline $\mathrm{La}$ & 15,7 & 9,2 & 13,5 & 29,9 & 30,7 & 34,7 \\
\hline $\mathrm{Ba}$ & 459,1 & 141,9 & 230,5 & 410,3 & 242,6 & 151,1 \\
\hline Cs & 3,9 & 4,4 & 7,3 & 14,8 & 11,5 & 8,9 \\
\hline
\end{tabular}

Tabla 3.-Concentraciones de Si, Al, Na y K (en ppm) en el filtrado después de 28 días de ataque y porcentaje de óxido liberado respecto al total de muestra (entre paréntesis)

\begin{tabular}{|c|c|c|c|c|c|}
\hline Muestra & Solución de ataque & $\mathrm{Si}$ & $\mathrm{Al}$ & $\mathrm{Na}$ & $\mathrm{K}$ \\
\hline AMR & $\mathrm{Ca}(\mathrm{OH}) 2$ & $16,0(0,05)$ & $1,1(0,00)$ & $47,5(2,52)$ & $4,3(0,19)$ \\
\hline AMR & $\mathrm{NaOH}$ & $595,0(1,79)$ & $522,2(3,91)$ & - & $125,8(5,50)$ \\
\hline DPB & $\mathrm{Ca}(\mathrm{OH}) 2$ & $22,1(0,06)$ & $0,0(0,00)$ & $42,6(2,57)$ & $30,3(0,43)$ \\
\hline DPB & $\mathrm{NaOH}$ & $189,5(0,52)$ & $215,1(1,99)$ & - & $199,1(2,80)$ \\
\hline
\end{tabular}



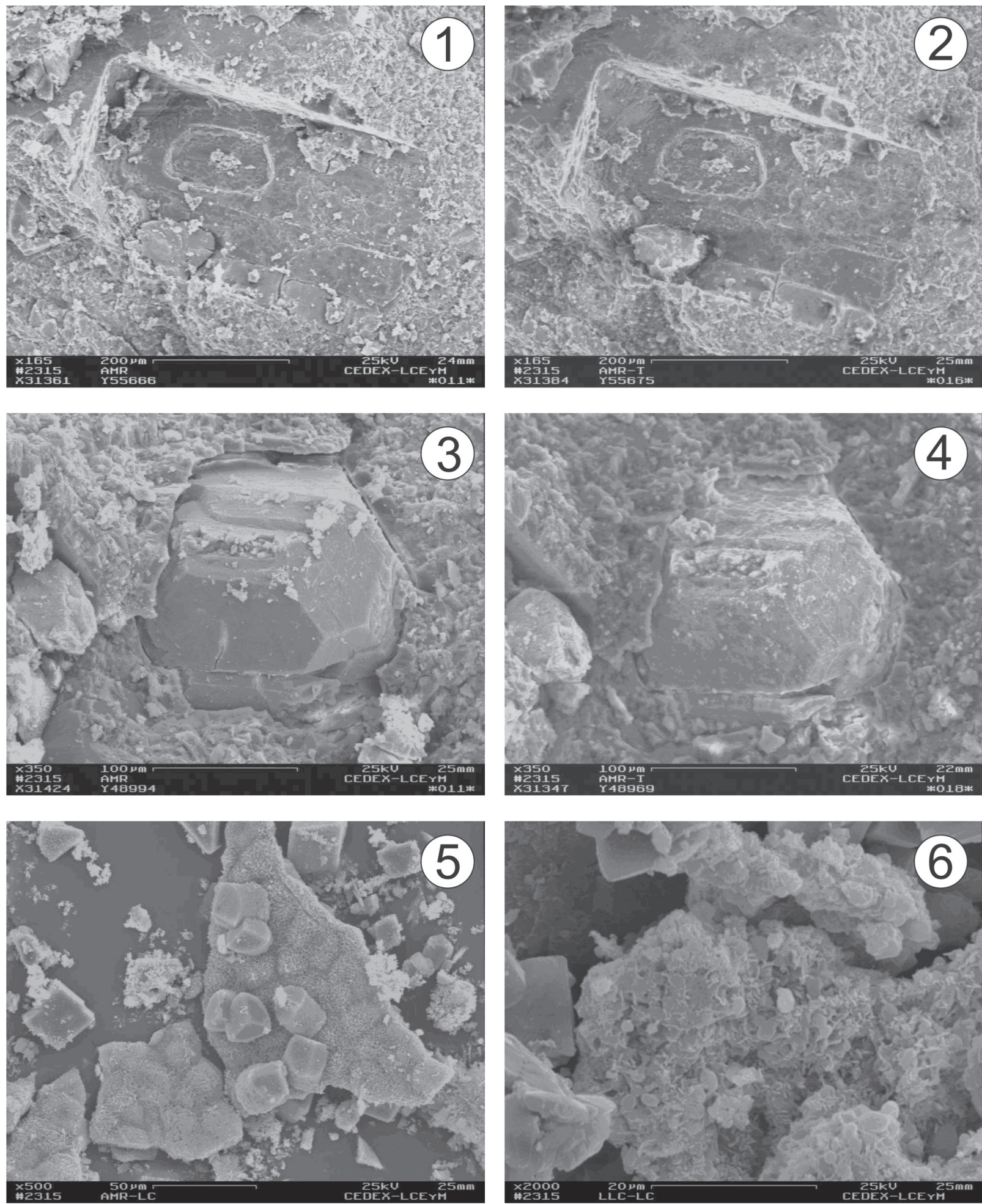

Fig. 3.-Reactividad superficial y residuo sólido. 1) Andesita piroxénica (AMR). Cristal de piroxeno antes de ser sometido a la solución de ataque; 2) Andesita piroxénica (AMR). Disolución parcial del cristal de piroxeno de la imagen anterior, después de ser sometido a la acción del líquido de ataque; 3) Andesita piroxénica (AMR). Cristal de anfíbol antes de ser sometido a la solución de ataque; 4) Andesita piroxénica (AMR). Disolución parcial del cristal de anfíbol de la imagen anterior, después de ser sometido a la acción del líquido de ataque; 5) Andesita piroxénica (AMR). Residuo sólido por evaporación. Geles rugosos; 6) Dacita anfibólica (DPB). Residuo sólido por evaporación. Productos polimorfos con textura alveolar. 

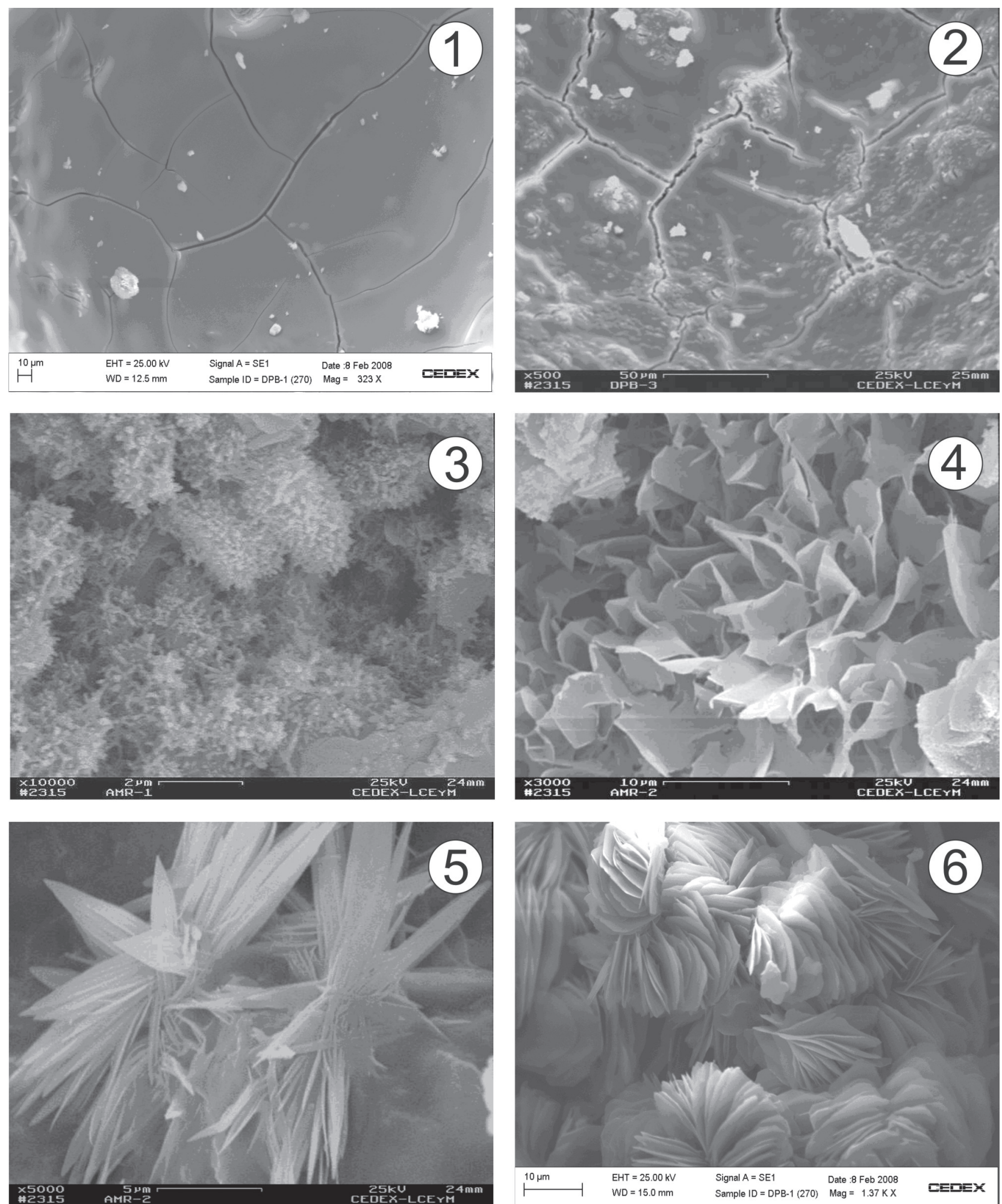

Fig. 4.-Superficie de fractura de las barras de mortero. 1) Dacita anfibólica (DPB). Barras de mortero. Geles lisos; 2) Dacita anfibólica (DPB). Barras de mortero. Geles rugosos; 3) Andesita piroxénica (AMR). Barras de mortero. Agregados botroidales; 4) Andesita piroxénica (AMR). Barras de mortero. Productos polimorfos con textura alveolar; 5) Andesita piroxénica (AMR). Barras de mortero. Productos polimorfos con textura acicular; 6) Dacita anfibólica (DPB). Barras de mortero. Productos polimorfos con textura en pétalos de rosa. 
Hay que señalar que si el ensayo se hubiese detenido a los 14 días, la expansión hubiera sido en ambas muestras inferior al $0,10 \%$, por lo que los áridos podrían haber sido considerados como no reactivos. Sin embargo, la expansión observada tanto a los 90 días $(0,26 \%)$ como a los 180 días $(0,40 \%)$ permite afirmar que el árido es reactivo y que el ensayo descrito en la norma UNE 146508 no es el adecuado para este tipo de rocas, como ocurre asimismo con algunos gneises y basaltos (Fournier \& Berubé, 1991 y Ponce \& Batic, 2006).

\section{Conclusiones}

Los tratamientos de las muestras con $\mathrm{NaOH}$ o $\mathrm{Ca}(\mathrm{OH})_{2}$ no producen importantes cambios ni en su composición mineralógica ni en su composición química.

Las concentraciones de $\mathrm{Si}$ y $\mathrm{Al}$ liberadas por los dos tipos de ataques se deben fundamentalmente a la disolución de los filosilicatos presentes (caolinita y esmectita), mientras que las de $\mathrm{Na}$ y $\mathrm{K}$ proceden de la disolución de los anfíboles y la biotita y sobre todo de la matriz.

El residuo sólido obtenido por evaporación hasta sequedad de los líquidos de ataque está constituido por geles y compuestos polimorfos, semejantes a los encontrados en un hormigón afectado por una reacción álcali-sílice.

La observación de la superficie de fractura de las barras de mortero indica que la expansión producida es debida a una reacción álcali-sílice.

La cinética de reacción álcali-sílice en las muestras estudiadas es muy lenta, por lo que la expansión detectada en las barras de mortero sólo sobrepasa los límites establecidos en la Normativa a partir de los noventa días.

\section{Referencias}

ACI Committee 201. (2007). ACI Manual of Concrete Practice, American Concrete Institute, 201.2R-1201.2R-41.

Chung, F.H. (1975). Quantitative interpretation of X-ray diffraction patters of mixtures. III Simultaneus determination of a set of reference intensities. Journal Applied Crystallography, 8: 17-19. doi:10.1107/S0021889875009454

Fournier, B. \& Berubé, M.A. (1991). Application of the NBRI accelerated mortar bar test to siliceous carbonatwe aggregates produced in the St. Lawrence Loowlands (Quebec, Canadá). Part 1. Influence of various parameters on the test results. Cement and Concrete Research, 21: 853-862. doi:10.1016/0008-8846(91)90180-P

López-Ruiz, J. \& Rodríguez Badiola, E. (1980). La región volcánica Neógena del sureste de España. Estudios Geológicos, 36: 5-63.

López-Ruiz, J. \& Wasserman, M.D. (1991). Relación entre la hidratación/desvitrificación y el $\delta^{18} \mathrm{O}$ en las rocas volcánicas neógenas del SE de España. Estudios Geológicos, 47: 3-11. doi:10.3989/egeol.91471-2404

López-Ruiz, J.; Cebriá, J.M. \& Doblas, M. (2002). Cenozoic volcanism I: the Iberian peninsula. In: The Geology of Spain (Gibbons, W. \& Moreno, T., eds.). Geological Society, London, 417-438.

Ponce, J.M. \& Batic, O.R. (2006). Different manifestations of the alkali-silica reaction in concrete according to the reaction kinetics of the reactive aggregate. Cement and Concrete Research, 36, 1148-1156. doi:10.1016/j.cemconres.2005.12.022

Soriano, J. (1987). Reactions d'interaction entre certains granulats et la phase instertitielle du beton. In: Pore Structure and Materials Properties. (Maso, J.C., ed.) Chapman \& Hall Eds. London, 25-32.

Way, S.J. \& Cole, W.F. (1982). Calcium Hydroxide attack on rocks. Cement and Concrete Research, 12: 611-617. doi:10.1016/0008-8846(82)90022-9

Recibido el 16 de noviembre de 2009 Aceptado el 15 de enero de 2010 Publicado online el 22 de abril de 2010 